\title{
Kernos
}

Revue internationale et pluridisciplinaire de religion grecque antique

5| 1992

Varia

\section{Langage orgiastique et glossolalie}

\section{Manuel García Teijeiro}

URL : http://journals.openedition.org/kernos/1048

DOI : 10.4000/kernos.1048

ISSN : 2034-7871

\section{Éditeur}

Centre international d'étude de la religion grecque antique

\section{Édition imprimée}

Date de publication : 1 janvier 1992

ISSN : 0776-3824

\section{Référence électronique}

Manuel García Teijeiro, «Langage orgiastique et glossolalie », Kernos [En ligne], 5 | 1992, mis en ligne le 19 avril 2011, consulté le 04 mai 2019. URL : http://journals.openedition.org/kernos/1048 ; DOI :

10.4000/kernos. 1048 
Kernos, 5 (1992), p. 59-69.

\section{LANGAGE ORGIASTIQUE ET GLOSSOLALIE}

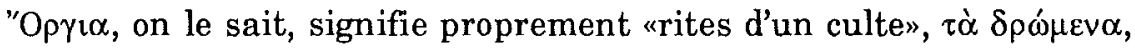

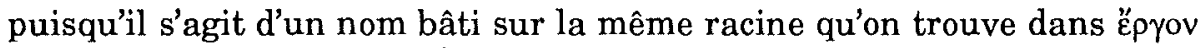
et les verbes $\varepsilon ̋ \delta \omega$ et $\dot{\rho}^{\prime} \zeta \omega^{1}$. Comme il est arrivé en partie à ces formes verbales, óp $ү \iota \alpha$ a donc pris une signification spécialisée ${ }^{2}$. Si nous voulions examiner le langage orgiastique dans ce sens, il faudrait prendre en considération la plupart des particularités de la langue religieuse : style solemnel, préservation d'archaïsmes, expressions symboliques, rejet de vocabulaire vulgaire, etc.

Mais le processus de spécialisation d'óp $\gamma\llcorner\alpha$ et de ses dérivés a continué. Le mot, sans perdre son acceptation générale, se dit surtout des cultes à mystères, notamment ceux que nous appelons extatiques et orgiastiques, dionysiaques en premier lieu, mais aussi des Cabires, de Sabazios, de la Cybèle phrygienne et de beaucoup d'autres. Voilà d'où vient la relation étymologique avec ópүฑ,, «agitation intérieure», défendue par les anciens grammairiens ${ }^{3}$.

Nous connaissons fort mal les mystères, mais il y a des indices qui montrent qu'on employait souvent un langage particulier.

Il n'est pas étonnant, bien sûr, que les membres d'une association secrète utilisent parmi eux un argot pour protéger les connaissances qu'ils partagent. Clément d'Alexandrie ${ }^{4}$ nous a transmis les notes d'un certain Epigénès ${ }^{5}$ sur le vocabulaire orphique. La clef de la signification de

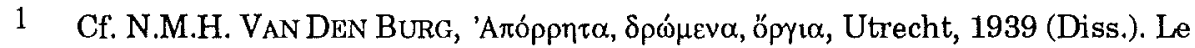
doute de P. Chantraine, La formation des noms en grec ancien, Paris, 1933, p. 55, ne semble pas justifié. Cf. aussi les dictionnaires étymologiques de H. Frisk et du même Chantraine. Probablement óp $\gamma$ ı $\alpha$ est une formation en - lov sur le radical verbal attesté par myc. wo-ze, wo-ze-e (*wrg-y-), «travailler

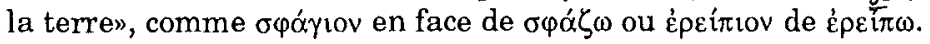

2 La spécialisation peut être déjà mycénienne, si wo-ro-ki-jo-ne-jo appartient à ce groupe et a des connotations religieuses dans les tablettes.

3 Servius, ad Aen., IV, 302 : sane sciendum orgia apud Graecos dici sacra omnia, sicut apud Latinos caerimoniae dicuntur, sed iam abusive sacra Liberi

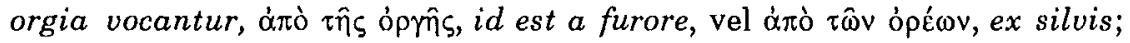
Etym. Magn., 629.

4 Strom., V, 8, 49 = Orphicorum fragmenta, 33 Kern. Cf. Th. HopfNeR, Griechisch-ägyptischer Offenbarungszauber, I, Amsterdam, 1974², p. 459 sq.

5 Il ressort du passage de Clément que Épigénès était antérieur à Callimaque. Il a vécu dans la première moitié du IVe siècle av. J.-C., s'il s'agit du disciple de Socrate cité par Platon et par Xénophon, comme l'a proposé I.M. LINFORTH, 
quelques termes dépend d'une comparaison implicitée entre le tissage et le labourage d'un champ. Ainsi, on appelait les charrues «navettes recour-

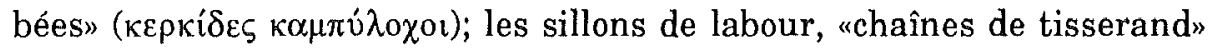

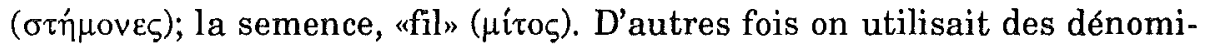

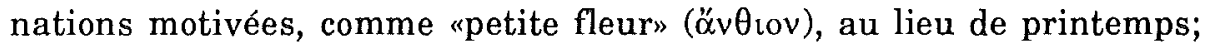

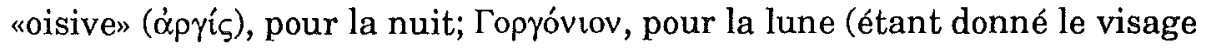
qu'on croit y voir); même de périphrases poétiques, qui forment de vraies kenningar : «larmes de Zeus», c'est la pluie; «Moïraï à la robe blanche», les phases de la pleine et de la nouvelle lune. Comme il arrivait au kenning islandais, le mécanisme de substitution pouvait se compliquer. Par exemple, les relations entre tisser et labourer, d'une part, et celle de labourer et de procréer. Ainsi, on employait le nom d'Aphrodite pour désigner le temps favorable pour les semailles (ó $\kappa \alpha \iota p o ̀ s ~ \kappa \alpha \theta^{\prime}$ öv $\delta \varepsilon \hat{\imath}$ $\sigma \pi \varepsilon i p \varepsilon(v)$, mais on allait plus loin. Un kantharos noir du Kabirion thébain, qui date probablement du Ve siècle, est décoré d'une scène qui attire l'attention : deux figures, nommées Mitos et Krateia, s'entretiennent ensemble. À leur côté se trouve une autre, plus petite, qui représente Protolaos, le premier homme, tandis qu'on trouve à gauche, couché sur un lit de table, Kabeiros, à l'aspect d'un Dionysos barbu, avec son $\pi \alpha \hat{\imath} \varsigma^{6}$. O. Kern allégua il y a longtemps cette peinture comme preuve d'une influence orphique sur le culte thébain 7 . Mitos et Krateia doivent être ici, en effet, des personnifications de la capacité procréatrice respective de l'homme et de la femme.

On trouve ailleurs des procédés similaires pour constituer un argot. Le même Clément l'indique pour les pythagoriciens, qui appelaient les planètes «chiens de Perséphone», et la mer «larmes de Kronos». Il y en a aussi dans les formes d'expression des sectes gnostiques ${ }^{8}$, dans les Decknamen de l'alchimie ${ }^{9}$ et de la botanique magique, par exemple dans

The Arts of Orpheus, Berkeley-Los Angeles, 1941, p. 114 sq. Cf. M.L. WEST, The Orphic Poems, Oxford, 1983, p. 9 sq., 248.

6 La scène est reproduite, p. ex., chez RosCHer, II, 2538, et chez M.P. NiLsSON, Geschichte d. gr. Religion, I, München, 1967³, Taf. 48, 1.

7 Die boiotischen Kabiren, î Hermes, 25 (1890), p. 1-16; RE, X, 1440 sq.; XV, 2216 sq. Cf. aussi B. HemB arg, Die Kabiren, Uppsala, 1950, p. 195 sq., 203 sq.; NiLsson, GGR ${ }^{3}$, I, p. 672; W. BurKerT, Homo necans, Berkeley, 1983, p. 246 sq., et Greek Religion, Oxford, 1985, p. 282.

8 Le chapitre Die Kunstsprache der Gnostiker, in R. REITZEnstein, Die hellenistischen Mysterienreligionen, Leipzig, $1927^{3}$, p. 393-401, est encore utile.

9 Cf., p. ex., M.P. Crosland, Historical Studies in the Language of Chemistry, London, 1962, p. 1-53. 
la liste que nous a préservée un papyrus magique de Leiden ${ }^{10}$, où on donne la signification de trente-sept de ces noms trompeurs : "larmes de babouin", c'est du jus d'aneth; "cœur de faucon» veut dire la moelle d'une certaine plante, l'armoise; etc. Le papyrus explique lui-même que, comme beaucoup de gens voulaient user de moyens magiques, les scribes des temples égyptiens imaginèrent ces dénominations bizarres, inspirées par les propriétés et "sympathies" des plantes et objets utilisés dans leurs enchantements ${ }^{11}$. Quand Alexarque, l'excentrique frère du roi de Macédoine Cassandre, voulut créer une langue artificielle pour sa «Ville céleste», l'Ouranopolis qu'il avait bâtie sur mont Athôs, il suivit une méthode semblable : aux noms communs du grec furent substituées des désignations qui faisaient allusion à quelque particularité de ce qui était nommé, ou bien à l'aide de la composition (il appelait, par exemple, le coq

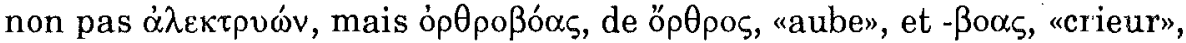
quelque chose comme le "Chanteclaire» du Roman de Renard), ou bien à l'aide de la dérivation (d́pyopís, "argentée», c'était, par exemple, le rıom de la drachme) ${ }^{12}$.

L'expression nouvelle est toujours, on le voit, un mot grec, mais c'est un vocable bizarre, qui remplace le nom habituel selon une motivation quelconque et ne peut être compris par celui qui le voit ou qui l'écoute, s'il ne connaît pas la clef. Il y a aussi sans doute une influence du goût pour les mots rares et pour le style recherché d'une part de la littérature alexandrine, comme les technopaegnia et l'Alexandra de Lycophron; mais le phénomène est beaucoup plus ancien que n'importe quelle mode littéraire. L'opposition entre une langue ordinaire, où la relation du signifiant avec le signifié est purement arbitraire, et une autre transparente, où les noms ne sont pas conventionnels, mais adaptés à la nature des choses ${ }^{13}$, apparaît depuis les premières spéculations sur le langage et laisse des

10 PGM, XII, 401-444.

11 Cf. Hopfner, OZ, I, p. 277 sq.; M.G. TeIJeiro, El vocabulario de la magia en los papiros griegos (à paraître dans Homenaje a J. Alsina).

12 Athenee, III, 98d-f (en citant Heracl. Lembos). Cf. Clem. Alex., Protr., IV, 54, 3 (il dépend d'Aristos de Salamine). Sur Alexarque, cf. O. Weinreich, Menekrates, Zeus und Salmoneus, Stuttgart, 1933 (= Religionsgeschichtliche Studien, Darmstadt, 1968, p. 299-434); W.W. TARN, Alexander the Great and the Unity of Mankind, London, 1933, p. 21-24, et Alexander the Great, II, Cambridge, 1948, p. 426 sq.; H.C. BALDRY, The Unity of Mankind in Greek Thought, Cambridge, 1965, p. 124 sq.; M.G. TEIJEIRo, Una lengua artificial en la Grecia helenistica, in RSEL, 11 (1981), p. 69-82.

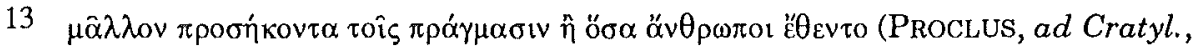
LI, p. 20, 15 Pasquali). 
traces dans les allusions à une langue des dieux, opposée à celle des hommes : chez Homère, dans l'épopée archaïque, chez Phérécyde, chez Pindare, chez Platon, chez les auteurs de comédies, chez Philoxène de Leucade et même dans un fragment orphique ou le carmen de viribus herbarum ${ }^{14}$. Comme la même opposition entre les deux langues est attestée dans un poème de l'Edda islandaise, l'Alvísmál, dans des textes sanscrits et hittites, et peut-être aussi dans la tradition irlandaise, elle remonte sans doute à l'époque indo-européenne et même au-delà 15 .

Mais les dieux pouvaient également parler une langue incompréhensible, formée par ces bruits de la nature que $O$. Rudolf appelait numinosen Urlauten, comme le murmure de l'eau ou les susurrements du vent. Souvent la langue des dieux se montre aussi dans les personnes atteintes de désordres mentaux qui articulent des phrases modulées que personne ne peut comprendre.

La littérature classique fait aussi mention de ces croyances. Chez

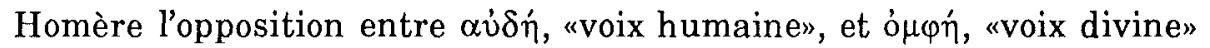
(mot qui, si l'on en juge d'après les formes germaniques de la même famille, évoque l'idée de la musique et du chant) est motivée probablement parce qu'on croit que les dieux parlent autrement que les humains, bien que dans les poèmes cela soit compris simplement comme une différence dans le ton de la voix ${ }^{16}$, étant donné la tendance constante à

14 Les témoignages, p. ex̦., chez M.L. WEst, dans son édition de la Théogonie d'HESIODE, ad v. 831.

15 Cf. H. GÜNTERT, Von der Sprache der Götter und Geister, Halle, 1921; A. Heubeck, Die homerische Göttersprache, in WJA, 4 (1949-1950), p. 197-218 (=Kleine Schriften, p. 94-115); J. FRIEDRICH, Göttersprache und Menschensprache im hethitischen Schriftum, in Festschrift A. Debruner, Berne, 1954, p. 135-139; R. LAZZERONI, Lingua degli dei e lingua degli uomini, in ASNP, 26 (1957), p. 1-25; C. WATKINS, Language of Gods and Language of Men : Remarks on Some I.E. Metalinguistic Traditions, in Myth and Law among the Indoeuropeans, Berkeley, 1970, p. 1-17; J. CLAY, The planktai and moly: Divine Naming and Knowing in Homer, in Hermes, 100 (1972), p. 127-131; J. CALderon Felices, Lengua de los dioses / lengua de los hombres, in Faventia, 4 (1982), p. 5-33; F.L. LETOUBLON, Les dieux et les hommes. La langue et sa référence dans l'Antiquité grecque et archaïque, in Language and Reality in Greek Philosophy, Athens, 1985, p. 92-99; F. BADER, La langue des dieux ou l'hermétisme des poètes indo-européens, Pisa, 1989; EAD., La langue des dieux, hermétisme et autobiographie, in $E C, 58$ (1990), p. 3-26; EAD., Autobiographie et héritage dans la langue des dieux : d'Homère à Hésiode et Pindare, in REG, 103 (1990), p. 383-408.

16 Cf. Od., V, 333-335, où l'on dit que Inô fut une femme mortelle, à la voix humaine, mais que après elle devint une déesse marine. Dans X, 136; XI, 8 et XII, 150 la «voix humaine» est attribuée à Circé; dans XII, 449, à Calypso. 
l'anthropomorphisme. Chez Hésiode, Th., 830-835, l'affreux Typhon a cent têtes qui émettent des voix que seuls les dieux peuvent comprendre, entre des mugissements, rugissements, aboiements et sifflements. Ajax, dans sa folie, prononce des injures si terribles, que seulement un démon pouvait les lui avoir apprises ${ }^{17}$.

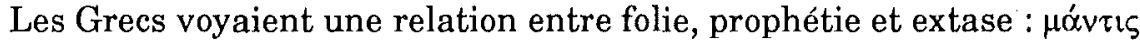
et $\mu \alpha \imath \alpha^{\prime} \zeta$ appartiennent à la même famille de mots que $\mu \alpha i v o \mu \alpha \imath$ et $\mu \alpha v i \alpha$. Les exclamations et les noms articulés à demi prenaient part aux fêtes

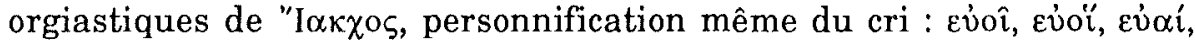

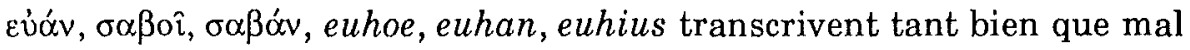
ces cris extatiques. Une curieuse inscription, gravée sur le bord d'un miroir, semble jouer avec des variations de ces cris :

\section{$\triangle H M \Omega N A \Sigma \Sigma A ~ \Lambda H N A I O E Y A I$ KAI $\Lambda$ HNAO $\triangle$ AMOK $\Lambda$ O EIAY 18}

La mantique extatique était sans doute remplie des phénomènes linguistiques anormaux. À défaut d'un terme plus approprié, nous parlons aujourd'hui de "glossolalie", un mot nouveau formé d'après l'expression

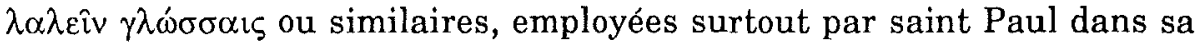
première épître aux Corinthiens ${ }^{19}$. Le sens du terme s'est élargi pour embrasser n'importe quelle manifestation incompréhensible du langage extatique. Il s'agit habituellement d'un phénomène résultant d'une intense émotion religieuse, aujourd'hui surtout dans la fête de la Pentecôte. La glossolalie a été très débattue et, sous sa modalité contemporaine, l'objet d'observations minutieuses, depuis les travaux classiques d'E. Lombard ${ }^{20}$ et d'E. Morsiman ${ }^{21}$ jusqu'aux études

Dans l'Odyssée, quand une divinité prend les traits d'un être humain, le poète

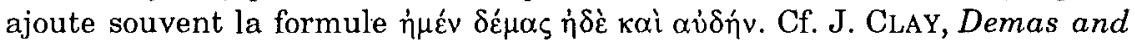
Aude : The Nature of Divine Transformation in Homer, in Hermes, 102 (1974), p. 129-136. Sur la relation entre la musique et la langue des dieux, cf. E.R. DonDs, The Greeks and the Irrational, Berkeley, 1951, p. 175, n. 119, avec la bibliographie.

17 SopH., Ajax, 243 sq.

18 Miroir en bronze, probablement de la fin du Ve siècle av. J.-C., trouvé dans un tombeau d'Olbia, en Scythie. Cf. F. TINNEFELD, in $Z P E, 38$ (1980), p. 70-71; M.L. West, The Orphic Poems, Oxford, 1983, p. 156 sq. Ici le cri dionysiaque a été altéré dans la seconde partie de la ligne, qui forme une phrase parallèle d'une façon caractéristique de ces inscriptions.

19 Cf. l'excellente synthèse de G. DAUTZENBERG, in RACh s.v. "glossolalie», avec les références.

20 De la glossolalie chez les premiers chrétiens et des phénomènes similaires, Lausanne, 1910.

21 Das Zungenreden geschichtlich und psychologisch untersucht, Tübingen, 1911. 
d'orientation socio-linguistique. Les psychologues et les psychiatres ${ }^{22}$ se sont aussi occupés du problème.

Il y a une typologie générale des phonations glossolaliques. Elles peuvent n'être qu'un murmure, un chuchotement confus, mais souvent les sons sont articulés et ils s'assemblent pour former des pseudo-phrases, qui donnent l'impression d'appartenir à une langue inconnue. Dans ce cas-là, les groupes phoniques tendent à avoir presque le double de syllabes qu'il est normal d'avoir dans la langue réelle ${ }^{23}$. Ces pseudo-phrases sont remplies de répétitions, des variations graduelles, d'allitérations, de rimes. L'ensemble peut se conformer à une mélodie. Le chant religieux allemand Laßt mich gehen fut «rendu» ainsi par un glossolale ${ }^{24}$ :

schua ea, schua ea,

o tschiri biro ti ra pea

akki lungo ta ri fungo

u li basa ti ra tungo

latschi bungo ti tu ta!

Parmi les allusions a des phénomènes glossolaliques dans la Grèce ancienne, on peut remarquer le passage de l'Hymne homérique à Apollon, 156-164, dans lequel est mentionné un $\mu \varepsilon ́ \gamma \alpha \theta \alpha \hat{v} \mu \alpha$, une grande merveille 25 :

Il y a le grand prodige dont la renommée ne périra jamais : les filles de Délos, servantes de l'Archer ! Après avoir célébré d'abord Apollon, puis ensuite Létô et l'archère Artemis, elles pensent aux hommes et aux femmes de jadis, et chantent un hymne à leur gloire, en charmant les familles humaines. Les langues de tous les hommes et leurs parlers

22 Le résumé de Dautzenberg (supra n. 19) contient une bonne bibliographie. Pour la glossolalie païenne, il est utile par la richesse de sa documentation, L. CaRlyle May, A Survey of Glossolalia and Related Phenomena in NonChristian Religion, in American Anthropologist, 58 (1956), p. 75-96. D'orientation socio-linguistique, W.J. SAMARIN, Glossolalia as Learned Behavior, in Canadian Journal of Theology, 15 (1969), p. 60-64; ID., Evolution in Glossolalic Private Language, in Anthropological Linguistics, 13 (1971), p. 55-67; ID., Tongues of Men and Angels: The Religious Language of Pentecostalism, New York, 1972.

23 E. Bryant-D. O'Donnel, A Phonemic Analysis of Nine Samples of Glossolalic Speech, in Psychonomic Science, 22 (1971), p. 82 (cité par L.J. HEIRMAN, in Mnemosyne, 28 [1975], p. 264, n. 27).

24 Cf. H. GüNTERT, Von der Sprache der Götter und Geister, Halle, 1921, p. 30 sq., avec les références.

25 H.J. TsChIEdel, Ein Pfingstwunder im Apollonhymnos (Hymn hom. Ap. 156 164) und Apg. 2, 1-13, in $Z R G G, 27$ (1975), p. 22-39. 
confus, elles savent les imiter; chacun jurerait que c'est lui-même qui parle, tant leur beau chant s'adapte avec fidélité !

(trad. J. Humbert, Collection des Universités de France)

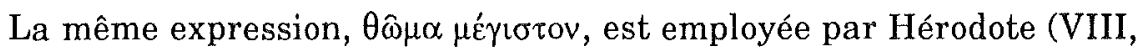
135) quand il raconte qu'un carien, appelé Mys, arriva au sanctuaire d'Apollon Ptôos de Béotie pour consulter l'oracle. Au sanctuaire, le $\pi \rho o ́ \mu \alpha v \tau \iota \varsigma$ commença soudain à parler une langue barbare, à l'étonnement des trois grecs qui accompagnaient Mys. Celui-ci cependant comprit très bien, puisque le dieu, à travers son prophète, avait parlé carien ${ }^{26}$.

Nous aimerions bien avoir la transcription phonétique d'une manifestation glossolalique de l'Antiquité, et nous aimerions bien aussi savoir qu'elle était exactement la fonction des $\pi \rho \circ \varphi \hat{\eta} \tau \alpha \iota$ et des prêtres médiateurs entre l'oracle et le consultant. On a proposé de voir un exemple de langage glossolalique dans les cris que pousse Cassandre en entrant dans le palais d'Agamemnon

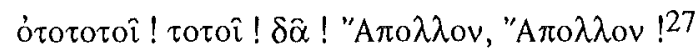

Les allitérations et les jeux de mots qui suivent mettraient en relief l'anomalie de son état.

Les voces magicae, si nombreuses dans les papyrus, les défixions et les talismans, apportent aussi un témoignage important ${ }^{28}$. Leur but est de contraindre le dieu ou le démon appelé à accomplir les ordres et les vœux du magicien. Ces mandats impératifs sont les vrais noms des divinités et des choses, groupés dans des formules irrésistibles. La doctrine de la parole magique est en rapport avec la croyance à une langue secrète, que parlent les dieux, formée par des sons très différents de ceux que prononcent les humains. Il ne s'agit pas d'une manifestation de langage extatique, mais il était très naturel qu'on établisse une relation entre prophétie, glossolalie et langue des dieux, puisque ces manifestations-là étaient considérées comme une preuve d'intervention divine. Clément d'Alexandrie en fait mention expressément, bien qu'en la rapportant à tort à Platon (Strom., I, 143, 1) :

26 Dans la brève mention de Pausanias (IX, 23, 6), Mys fait la consultation en carien. Dans le récit d'Hérodote, par contre, il est clair qu'elle fut formulée en grec.

27 Ag., 1072 sq. = 1076 sq. Cf. L.J. HeIRMAN, Kassandra's Glossolalia, in Mnemosyne, 28 (1975), p. 257-267.

28 M.G. TeIJeiro, Recursos fonéticos y recursos gráficos en los textos magicos griegos, in RSEL, 19 (1989), p. 233-249. 
Platon assigne aussi aux dieux une langue propre. Il justifie cette opinion surtout par les songes et les formules des oracles, mais aussi par les possédés, qui n'émettent pas plus leur propre voix ni leur propre langue, mais celles des divinités agissant à travers eux.

Étant donné cela, rien d'étonnant à ce que les voces magicae gardent des ressemblances avec les phonations extatiques. Les papyrus préviennent souvent sur l'opportunité d'émettre à des endroits fixes de la formule

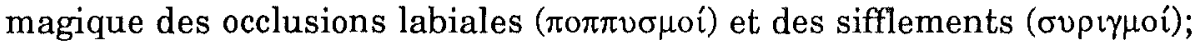
d'autres fois, il est question d'imitations de cris des animaux. Une invocation à la lune ${ }^{29}$, par exemple, dénombre quatorze caractéristiques phoniques du nom de la déesse : d'abord, silence ( $\sigma$ เүฑ), ensuite, occlusion

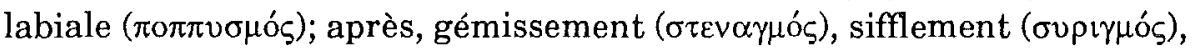
hurlement (ỏ̇o $\lambda v \gamma \mu o ́ s)$, etc.

Le «Huitième livre de Moïse» donne vers la fin ${ }^{30}$ ces instructions :

Expire, inspire, remplis tes poumons, $\varepsilon_{l} \cdot \alpha_{l} \cdot \alpha_{\alpha l}$. Reprends haleine et

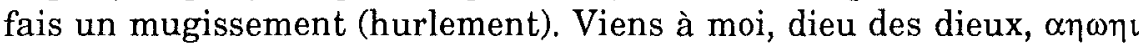
$\eta \iota 1 \alpha \omega$ oเ $\omega \tau \kappa$. Inspire profondément, prends de l'air avec les yeux fermés; meugle de toutes tes forces; ensuite pousse un gémissement en exhalant l'air.

Avec ses sons inarticulés, de phonétique purement expressive, on peut

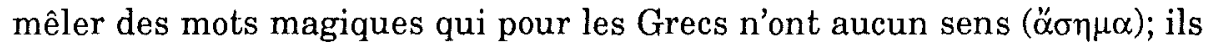
semblent appartenir à une langue étrangère $(\beta \alpha \rho \beta \alpha \rho \imath \alpha \alpha)$, et ils ont beaucoup de syllabes ( $\pi$ o $\lambda v \sigma v ́ \lambda \lambda \alpha \beta \alpha$ ). Entre eux il y a sans doute des mots égyptiens, juifs, syriens... Mais ce qui nous intéresse à présent, c'est qu'ils s'assemblent pour former des formules, qui, comme les manifestations glossolaliques, ont une structure caractéristique, une sorte de psalmodie, émise dans un long et lent murmure, comme les textes l'indiquent ${ }^{31}$. Parfois aussi la prononciation devait être très confuse, puisqu'il fallait mettre un doigt sous la langue. Les combinaisons des sons transgressent souvent les lois phonétiques du grec. On trouve des séries de syllabes comme форфор..., sans la dissimilation régulière (loi de Grassmann); des consonnes à la fin d'un mot que le grec n'admet pas; des noms ou groupes

29 PGM, VII, 765-779.

30 PGM, XIII, 941-946.

31 Ov., Mét., VII, 251; STACE, Theb., IV, 418; Firm. Mat., Err., 22,1. Cf. L. BALDINI MOSCARDI, Murmur» nella terminologia magica, in SIFC, 48 (1976), p. 254-262. Les sources grecques parlent d'une prononciation relâchée : $P G M$,

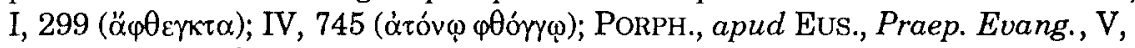

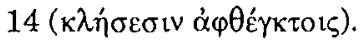


de mots qui peuvent être lus indifféremment de gauche à droite ou de droite à gauche $(\pi \alpha \lambda i ́ v \delta \rho \circ o l)$. Mais c'est surtout par l'ordre anormal des syllabes et des sons qu'on arrive à ces baragouins si caractéristiques des formules magiques. Dans le grand papyrus magique de Paris, par exemple, après un hymne maléfique à Sélènè, on appelle la déesse de ses vrais noms (PGM, IV, 2348-2355):

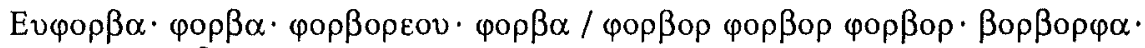
$\eta \varphi \varphi о \rho \cdot / \varphi о \rho \beta \alpha i \omega . .$.

La série se prolonge encore avec des variations monotones sur les syllabes $\varphi \circ \rho \beta \alpha$, comme s'il n'était pas possible pour le magicien de s'en débarrasser ${ }^{32}$. Qu'on compare la formule :

$\alpha \lambda \lambda \alpha \lambda \alpha \lambda \alpha \cdot \alpha \lambda \lambda \alpha \lambda \alpha \lambda \alpha \cdot \sigma \alpha \nu \tau \alpha \lambda \alpha \lambda \alpha \cdot \tau \alpha \lambda \alpha \lambda \alpha$

d'une invocation à Apollon ( $P G M$, II, 4), avec cette phrase glossolalique, prise par un témoin à $O \operatorname{slo}^{33}$ :

sangala, singala, sing, sing,

mangala, mangala, mang, mang, mang.

Les combinaisons vocaliques sont particulièrement fréquentes dans les documents magiques. Elles ont des connotations mystiques, comme des variations du grand nom de Dieu, I $\alpha \omega$, comme des symboles des sept planètes, etc. Il est intéressant qu'on opère précisément avec les sept signes de l'alphabet grec; pas avec les phonèmes de la langue, qui avaient été douze en Attique (sept voyelles longues et cinq brèves) et ils étaient six à l'époque des papyrus ${ }^{34}$. Avec les lettres on peut écrire n'importe quel nom, c'est pourquoi elles renferment l'univers tout entier.

On peut utiliser le rapport entre lettres et langages mystérieux avec un passage d'Apollodore de Corcyre ${ }^{35}$. Il raconte que le devin Branchos libéra Milet d'une plaie en aspergeant les habitants avec des rameaux de laurier et en chantant en l'honneur d'Apollon et d'Artémis; le peuple répondait avec le refrain :

$\beta \varepsilon \delta v \zeta \alpha \psi \chi \theta \omega \mu \pi \lambda \eta \kappa \tau \rho \circ \nu \sigma \varphi \imath \gamma \xi$ $\kappa v \alpha \xi \zeta \beta \imath \chi \theta v \pi \tau \varsigma \varphi \lambda \varepsilon \gamma \mu \mathrm{o} \delta \rho \omega \psi$

32 F. Dornseiff, Das Alphabet in Mystik und Magie, Leipzig-Berlin, 1925², p. 55.

33 Apud H. GÜNTERT, op. cit., p. 30.

34 Quand la marque de quantité cessa d'être pertinente et tandis que subsista la distinction entre /y/ et $/ \mathrm{i} /$.

35 Strom., V, 8, 48, 4. 


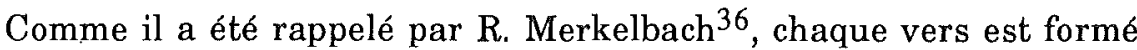
par les vingt-quatre lettres de l'alphabet ionien, groupées dans des mots capricieux. On proposa dans l'Antiquité beaucoup d'explications pour ces pseudo-mots : pour les unes, des interprétations mystiques; pour les autres, des identifications avec les quatre éléments de la nature, suggé-

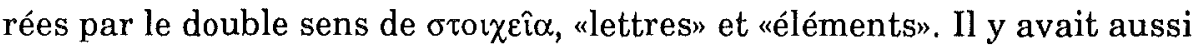
une autre formule, composée également par les vingt-quatre lettres de l'alphabet, groupées dans ce cas dans les «mots" :

$\mu \alpha \rho \pi \tau \varepsilon, \sigma \varphi \imath \gamma \xi, \kappa \lambda \omega \psi, \zeta \beta v \chi \theta \eta \delta o v$

Clément d'Alexandrie recueille ces curiosités dans le huitième chapitre du cinquième livre de ses Stromata. Il veut montrer qu'il n'y a rien d'étonnant dans l'interprétation allégorique de la Bible, puisque aussi bien les Barbares que les Grecs se sont servis souvent du langage symbo-

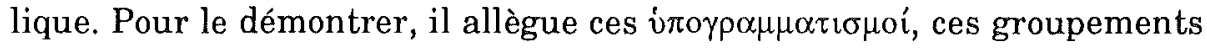
de lettres que nous venons de voir. Il cite aussi les interprétations d'un Pythagoricien, Androkydès, pour quelques autres mots mystérieux, et celles d'Épigénès pour les expressions orphiques dont nous avons fait mention au début de notre exposé, à propos des argots des sectes. Il s'agissait, comme nous l'avons déjà vu, de périphrases poétiques et de substitutions. Si l'on examine le chapitre de Clément, on voit que les mots étranges qui ne sont pas grecs reçoivent une explication semblable, motivée, par exemple, par une étymologie fantaisiste. Implicitement on trouve l'idée qu'il s'agit de mots élevés, au sens profond, que l'investigation peut dégager. La notion opposée est celle qui conçoit les formules magiques, les voix des possédés et le langage mystique, tout à fait différent de celui des hommes, au-delà de notre compréhension. Les deux notions se mêlent souvent. L'une et l'autre se trouvent dans les traditions qui parlent d'une langue divine. La première prédomine dans la littérature et dans les disputes sur l'origine du langage. La seconde est plus populaire, elle figure très souvent dans les mots mystérieux des enchantements. Nous croyons que la forme de ces vocables fantastiques fut influencée par les manifestations de langage extatique que nous appelons glossolalie. On a même remarqué certaines similitudes entre les

36 Weisse $\kappa v \alpha \xi \zeta \beta_{\mathrm{t}-M i l c h}$ (zu Thespis 1 F 4 Snell), in ZPE, 61 (1985), p. 293-296. Cf. aussi F. DoRNSEIF, Das Alphabet..., p. 69 sq. (plus d'exemples d'utilisation de l'alphabet tout entier); HopFNER, $O Z$, I, p. 458 sq. 
caractéristiques du langage des schizophrènes et les ressources de la langue poétique ${ }^{37}$.

Nul homme dans son bon sens ne parvient à la divination divine et véridique. Mais il faut que la force de son esprit soit entravée par le sommeil ou la maladie, ou qu'il l'ait déviée par quelque crise d'enthousiasme

disait Platon dans un passage très connu sur l'origine divine de la divination $^{38}$.

Departemento de Filología Clásica

Manuel GARCIA TEIJEIRO

Facultad de Filosofía y Letras

Universidad de Valladolid

E - 47002 VALLADOLID

37 Cf., p. ex., l'intéressante monographie de A. Метте, Ueber Beziehungen zwischen Spracheigentümlichkeiten Schizophrener und dichterischer Produktion, Dessau, 1928.

38 Tim., 71e (trad. d'A. Rivaud, Collection des Universités de France). 